\title{
Effect of Nintendo WII Brain Training Games on Perceptual Speed, Working Memory and Spatial Orientation in Young Adults
}

\author{
Garima Wadhwa ${ }^{1}$, Shefali Walia ${ }^{2}$ \\ ${ }^{1}$ Department of Rehabilitation Services for Children, Delhi Council for Child Welfare, New Delhi, India \\ ${ }^{2}$ Department of Neurology-Physiotherapy, Indian Spinal Injuries Centre-Institute of Rehabilitation Sciences, New Delhi, India
}

Email address:

garimawdhw@gmail.com (G. Wadhwa),shef0604@gmail.com (S. Walia)

\section{To cite this article:}

Garima Wadhwa, Shefali Walia. Effect of Nintendo WII Brain Training Games on Perceptual Speed, Working Memory and Spatial Orientation in Young Adults. International Journal of Neurologic Physical Therapy. Vol. 4, No. 1, 2018, pp. 7-15.

doi: 10.11648/j.ijnpt.20180401.12

Received: December 17, 2017; Accepted: January 5, 2018; Published: January 19, 2018

\begin{abstract}
Young adults are presumed to be healthy but the available evidences indicates a gradual decline of about $-0.02 \mathrm{SD}$ of fluid abilities starting in third decade of life. Brain training games are the new emerging methods for various domains of cognitive training. The purpose of this study is to identify the effectiveness of brain training games for perceptual speed, working memory and spatial orientation in young adults. Forty-eight healthy young adults who were willing to participate in the study were recruited. After taking informed consent, participants were assigned to either of two groups by lottery method (Nintendo WII brain training group, Nintendo WII Exergame group). Participants in both the groups played their games for about 30-45 minutes per day, 5 days a week for 4 weeks. Measures of the cognitive function the Number Comparison Test, Trail Making Test, Backward Digit Span Test and Perspective Taking Spatial Orientation Test were conducted before and after intervention. The comparison of pre-intervention result of the two groups revealed no significant difference in all variables, while significant improvements were observed in all measuring variables of the two groups when comparing their pre and post treatment mean values. Furthermore, the Nintendo brain training groups showed a significant improvement in Number Comparison Test $(\mathrm{p}=0.02)$, Trail Making Test Part A $(\mathrm{p}=0.03)$ And $\mathrm{B}(\mathrm{p}=0.05)$, Backward Digit Span Score Measure $(\mathrm{P}=0.02)$ and Perspective Taking Spatial Orientation Test $(\mathrm{p}=0.01)$ as compared to the Nintendo WII exergaming group. However no significant difference was noted on Backward Digit Span Length Measure. Nintendo WII brain training games are more efficient in improving perceptual speed, working memory and spatial orientation in young adults than Nintendo WII exergames.
\end{abstract}

Keywords: Young Adults, Perceptual Speed, Working Memory, Spatial Orientation, Nintendo WII, Brain Training Games, Exergames

\section{Introduction}

Young adults form precious human resources in every country with the age spanning 18-29 [1]. They are presumed to be healthy but the available evidences indicates a gradual decline of about $-0.02 \mathrm{SD}$ of fluid abilities starting in third decade of life $[2,3,4,5]$. This fluid intelligence (ability) is reportedly critical for wide varieties of cognitive tasks and is one of the most important factors in learning [3, 6]. The domains of fluid intelligence underpin every day multitasking, helps in understanding complex relationship, memorizing and solving new problems, behavioral regulation and adaptive functioning [7]. Perceptual speed for instance, in an ability to rapidly and accurately search, compare identify elements presented side by side or separated in visual field $[8,9]$. Working memory is the limited capacity storage system involved in maintenance and manipulation of information over short period of time [10,11]. Furthermore, spatial orientation helps to find out direction and location of one object with perspectives of the observer $[11,12]$

Sleep deprivation, physical activity, mental illness and 
stress are some of the important lifestyle factors that have been reported to adversely affect the cognitive abilities in young adult $[13,14,15]$. In addition to the lifestyle factors, there are neurobiological volumetric changes in prefrontal cortex, stratium and neurochemical reduction in dopamine transmission in early 20's that have been accounted for reduced cognitive flexibility, working memory and strategic encoding of time linked episodic memories [7].

In the new advance modern era of virtual reality based computer generated environment, the videogames to train the brain are being developed which engages individual to a real world situations and allows individual to do multitasking, help faster switching between the tasks without confusion. The most available videogame consoles are Nintendo WII, Microsoft Xbox kinetic and Dance dance revolution [16]

Nintendo WII with its incorporated innovative features of wireless controller, sensors detecting motion and rotation have become a familiar console in a family oriented gaming facilitating a wide range of physical experience with Nintendo WII exergames and brain training methods than a traditional form of a game play [16].

The number of studies attempted to show the effectiveness of brain training and exergames in improving some of the cognitive domains were reported on children, young adults, middle age, older adults but, no study has yet reported the use of Nintendo WII brain training games for perceptual speed, working memory and spatial orientation in young adults compared with the NintendoWII exergame [9, 17, 18, 19]. Hence, the purpose of study is to identify the effectiveness of brain training games for perceptual speed, working memory and spatial orientation in young adults.

\section{Methods}

The study was approved by Institution Research Review Committee and Ethical Committee. A sample of convenience of 48 healthy young adults who met inclusion criteria and were willing to participate and ready to attend exercise program were selected from India Spinal Injury Centre Hospital, New Delhi. The subjects included Hospital staff, students and patient's attendant.

\subsection{Study Design}

Pre test-Post test Experimental Design. The study was approved by Institution Research Review Committee and Ethical Committee. A sample of convenience of 48 healthy young adults who met inclusion criteria willing to participate and were ready to attend exercise

\subsection{Inclusion Criteria}

1. Healthy young adults of age between 18-29 [1]

2. Able to read and understand English and should have basic mathematical skills

3. Should not have any previous experience of Nintendo WII games [16]
4. Should be right handed [19]

5. $\mathrm{MOCA}>26$ [20.21]

\subsection{Exclusion Criteria}

1. Past episodes or history of unusual response to flashing light $[16,23]$

2. Any injury, fracture in upper limb and lower limb.

3. Any uncorrected hearing or visual problem [17]

4. Any neurological problem

5. Any history of sleeping disorder and bad sleep hygiene.

6. Using medication known to interfere cognitive function (benzodiazepine) [19]

7. Subject scoring less than 14 on Beck depression inventory II [22]

Demographic data including age, gender, education level MOCA score and sleep hours were noted (table 1). A thorough evaluation was done along with base line assessment.

\subsection{Overview of Cognitive Function Measure}

Number Comparison Test (NCT) [24, 25] - the subjects were to search the numbers and put ' $\mathrm{x}$ ' on non-matched numbers on a line present between them in a given period of one and half minutes for each two parts of this test. Their score was the total numbers marked correctly minus the number marked incorrectly.

Trail Making Test part A (TMT A) [26] - subjects were to connect the numbers in ascending order from 1-25 as quickly as possible without lifting pencil from paper.

Trail Making Test part B (TMT B)- subjects were to draw a continuous line connecting circles in ascending order, alternating between (number 1 through 12) and letter (A to L) as quickly as possible without lifting pencil from the paper. The score for trail making part A and B was the time taken to complete test in seconds

Backward Digit Span Test (BDS) [28] - Participants answer numbers in reverse order of that presented aloud by the examiner beginning with simplest level at two digit sequence and was increased one by one until the two consecutive failure of the same span length was noted. The primary measure of this test is the raw scores and the longest span length achieved.

Perspective Taking Spatial Orientation test (PTSOT) [29]The 12 item test was presented with the picture of 7 objects on top half of the paper and subjects were to imagine themselves standing at one object, facing the other, the task is to mark the direction of the third object on a circle presented on bottom half at the paper. The score was mean absolute deviation in degrees between participant's response and the correct direction of the target.

The comparison of pre intervention variables between group 1 and 2 were made (table 2). All the outcome variables were used in the study after permission from their respective founders. 
Table 1. Comparison of Demographic Data between Group 1 and 2.

\begin{tabular}{lllllll}
\hline \multirow{2}{*}{ Demographic profile } & Group 1 (N=24) & & Group 2 (N=23) & & \multirow{2}{*}{ t value } \\
\cline { 2 - 5 } & Mean & SD & Mean & SD & $0.44^{\mathrm{NS}}$ & 0.65 \\
Age (years) & 23.96 & 2.66 & 23.63 & 1.99 & $-0.25^{\mathrm{NS}}$ & 0.80 \\
Education (years) & 15.63 & 1.55 & 15.74 & 1.54 & $-0.67^{\mathrm{NS}}$ & 0.50 \\
Sleep Hours (hours) & 7 & 0.48 & 7.11 & 0.60 & $-0.94^{\mathrm{NS}}$ & 0.35 \\
MOCA & 27.29 & 0.95 & 27.57 & 1.03 & \\
Gender & $12 \mathrm{~F}, 12 \mathrm{M}$ & & $13 \mathrm{~F}, 10 \mathrm{M}$ & & \\
\hline
\end{tabular}

SD: Standard Deviation, p-Value: Level of significance, NS: Non-Significant.

Table 2. Comparison of Pre intervention measures between Group 1 and Group 2.

\begin{tabular}{|c|c|c|c|c|c|c|}
\hline \multirow{2}{*}{ Variables } & \multicolumn{2}{|c|}{ Pre - intervention: Group $1(n=24)$} & \multicolumn{2}{|c|}{ Pre - intervention: Group $2(n=23)$} & \multirow{2}{*}{ T value } & \multirow{2}{*}{ P value } \\
\hline & Mean & SD & Mean & SD & & \\
\hline NCT (score) & 23.50 & 7.59 & 24.17 & 7.94 & $-0.29^{\mathrm{NS}}$ & 0.76 \\
\hline TMT-A (time in sec.) & 29.69 & 6.80 & 29.25 & 10.42 & $0.16^{\mathrm{NS}}$ & 0.86 \\
\hline TMT-B (time in sec.) & 55.65 & 18.89 & 55.94 & 25.53 & $-0.04^{\mathrm{NS}}$ & 0.96 \\
\hline BDS Score & 5.50 & 1.79 & 5.70 & 1.94 & $-0.35^{\mathrm{NS}}$ & 0.72 \\
\hline BDS length & 3.63 & 1.01 & 3.57 & 1.16 & $0.18^{\mathrm{NS}}$ & 0.85 \\
\hline PTSOT (in degrees) & 67.24 & 42.54 & 66.77 & 48.98 & $0.03^{\mathrm{NS}}$ & 0.97 \\
\hline
\end{tabular}

SD: Standard Deviation, P-Value: Level of significance, NS: Non-significant.

\subsection{Overview of the Intervention}

After the baseline assessment measures the subjects were allocated to either of the two groups by the lottery method.

\subsubsection{Nintendo WII Brain Training Games}

Subjects were trained on 10 trail practice sequence each of the following: 5 mini games at their own speed from Nintendo WII big brain academy 1) Whack match (figure 1)the subjects were to grab the defined objects as quickly as possible when it appears on screen. 2) Covered Cage- the subjects were to memorize the position of birds when the jumble. 3) Balloon Burst- the subjects were to pop (burst) the correct order of balloons starting from lowest to highest as quickly as possible. 4) Color Count- the subjects were to maintain the track of different color balls (blue and red) coming inside basket. 5) Train Turn- the subjects were to figure out the correct pathway for train destination.

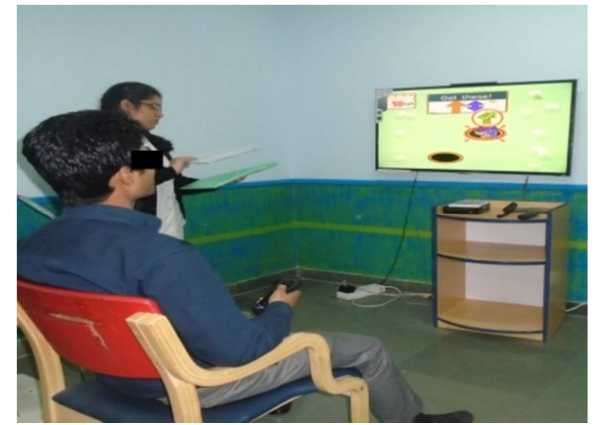

Figure 1. Subject playing Whack Match game from Nintendo WII Big Brain Academy.

\subsubsection{Nintendo WII Exergame Group}

Subjects were trained on the following exergames from Nintendo WII sports and WII Fit CD. 1) Soccer Header (figure 2) - subjects were to quickly shift the weight side to side by leaning left to right to block the soccer balls as they came fling to the head and not to hit shoe and other obstacles. 2) Ski Slalom - the subjects were to 'slalom' by shifting their weight to alternate between red and blue doors as quickly as possible. 3) Ski Jump- the subjects were to squat with bend knee until the point came where they have to extend quickly and hold still to the end- point. 4) Hula Hoop- the score was the number of successful rotations of the hoop in 70 seconds 5) Tennis- the subjects played single set game of tennis on single player mode. 6) Bowling- subjects were required to complete ten games for each bowling session.

The training took approx 30-40 minutes every session and was given for 5 times per week for a period of 4 weeks.

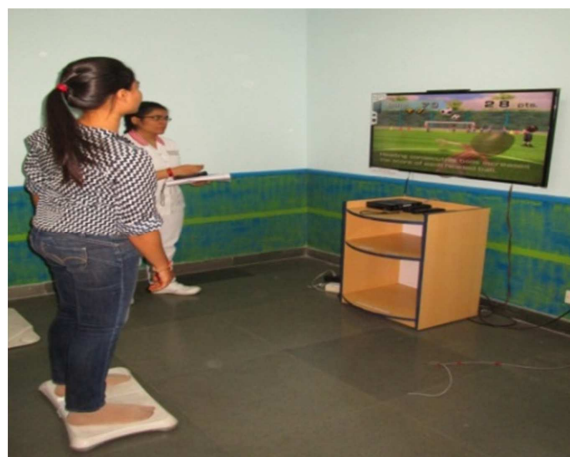

Figure 2. Subject playing Soccer Header game from WII-Fit games using WII- Fit balance board.

\subsection{Data Analysis}

Statistics were performed using SPSS (Statistical package for social science, version 21). The scores of Number Comparison Test, Trail making test part A and B, Backward Digit Span Test and Perspective Taking Spatial Orientation Test were analyzed for within group comparison using paired t test and between group comparisons were done using independent $t$ test. 


\section{Results}

Group 1 consisted of 12 females and 12 males; with the mean age $23.96 \pm 2.66$ years, mean education $15.63 \pm 1.55$ years, mean sleep hours $7 \pm 0.48$ hours and with the mean MOCA score of 27.29 \pm 0.95 . Group 2 consisted of 13 females and 10 males, with the mean age $23.63 \pm 1.99$ years, mean education $15.74 \pm 1.54$ years, mean sleep hours7.11 \pm 0.60 hours and with the mean MOCA score of $27.57 \pm 1.03$ (table1) one subject dropped out from group 2.

The results showed no significant difference in pre intervention scores of Number Comparison test (NCT) $(\mathrm{t}=$ $0.29, \mathrm{p}=0.76)$, Trail Making Test (TMT A) $(\mathrm{t}=0.16, \mathrm{p}=0.86)$ Trail making test part B (TMTB) $(-0.04, p=0.96)$, Backward digit span score $(\mathrm{t}=-0.35, \mathrm{p}=0.72)$, Backward digit span length measure $(t=0.18, p=0.52)$ and Perspective Taking Spatial Orientation Test (PTSOT) $(\mathrm{t}=-0.35, \mathrm{p}=0.72)$ between group 1 and group 2. (table 2)

The comparison of pre- intervention scores and postintervention scores of Group 1 showed a significant difference in Number Comparison Test $(\mathrm{t}=-10.70, \mathrm{p}=0.00)$, Trail Making Test part A $(\mathrm{t}=8.58, \mathrm{p}=0.00)$ Trail Making Test part $\mathrm{B}(\mathrm{t}=7.55, \mathrm{p}=0.00)$ Backward Digit Span Test Score $(\mathrm{t}=-15.02, \mathrm{p}=0.00)$, Backward Digit Span Length $(\mathrm{t}$ $=-6.82, \mathrm{p}=0.00)$ and in Perspective Taking Spatial Orientation Test $(\mathrm{t}=6.38, \mathrm{p}=0.00)($ table 3$)$.

The comparison of pre- intervention scores and postintervention scores of Group 2, also showed a significant difference in Number Comparison Test $(\mathrm{t}=-7.47, \mathrm{p}=0.00)$, Trail Making Test part A $(\mathrm{t}=7.41, \mathrm{p}=0.00)$ Trail Making Test part B $(\mathrm{t}=5.79, \mathrm{p}=0.00)$ Backward Digit Span Test Score $(\mathrm{t}=-10.00, \mathrm{p}=0.00)$, Backward Digit Span Length $(\mathrm{t}$ $=-6.49, \mathrm{p}=0.00)$ and in Perspective Taking Spatial Orientation Test $(\mathrm{t}=5.57, \mathrm{p}=0.00)$ (table 4).

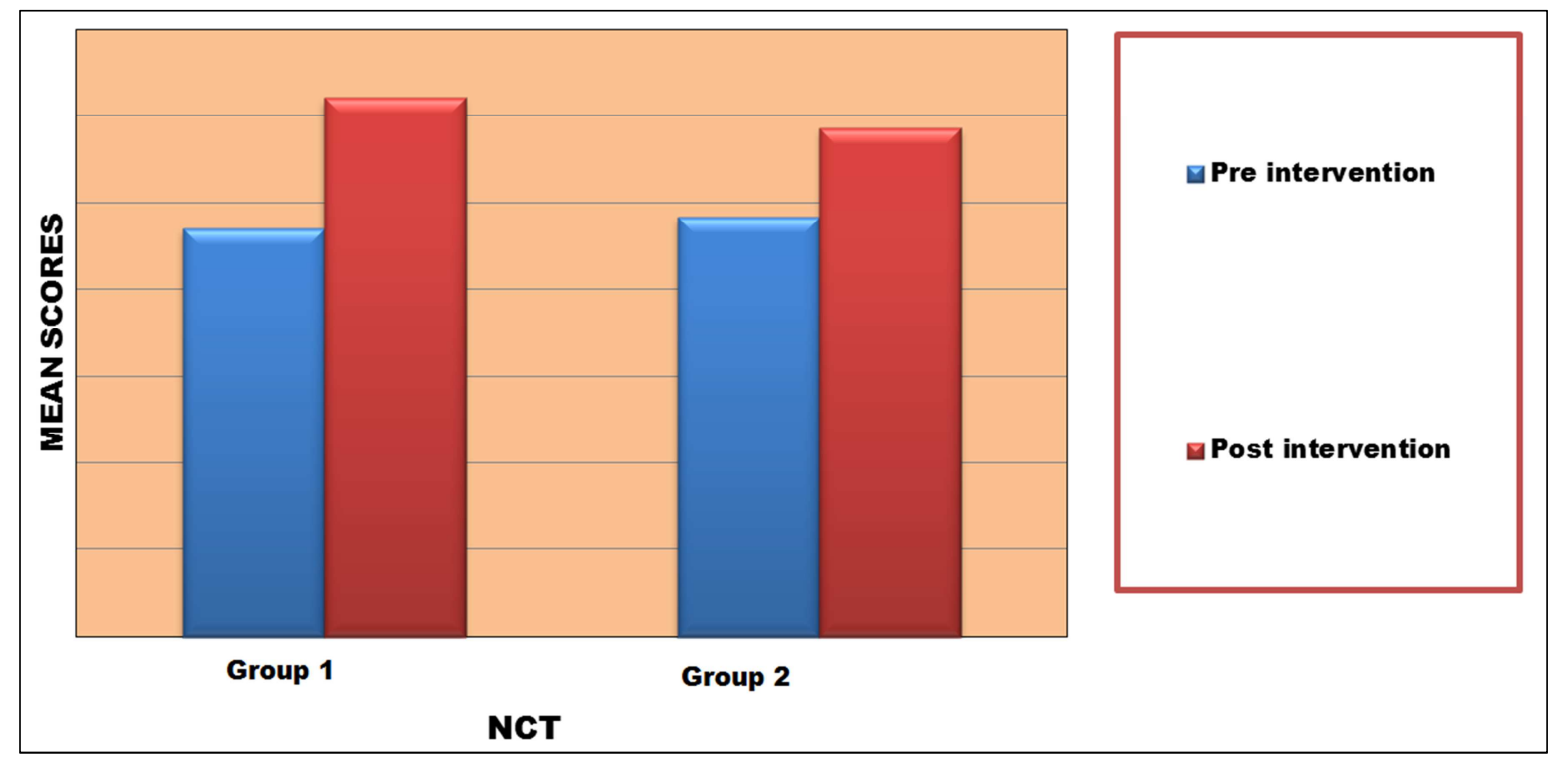

Figure 3. Comparison of Pre intervention and Post intervention in Number Comparison Test (NCT) of Group 1 and Group 2.

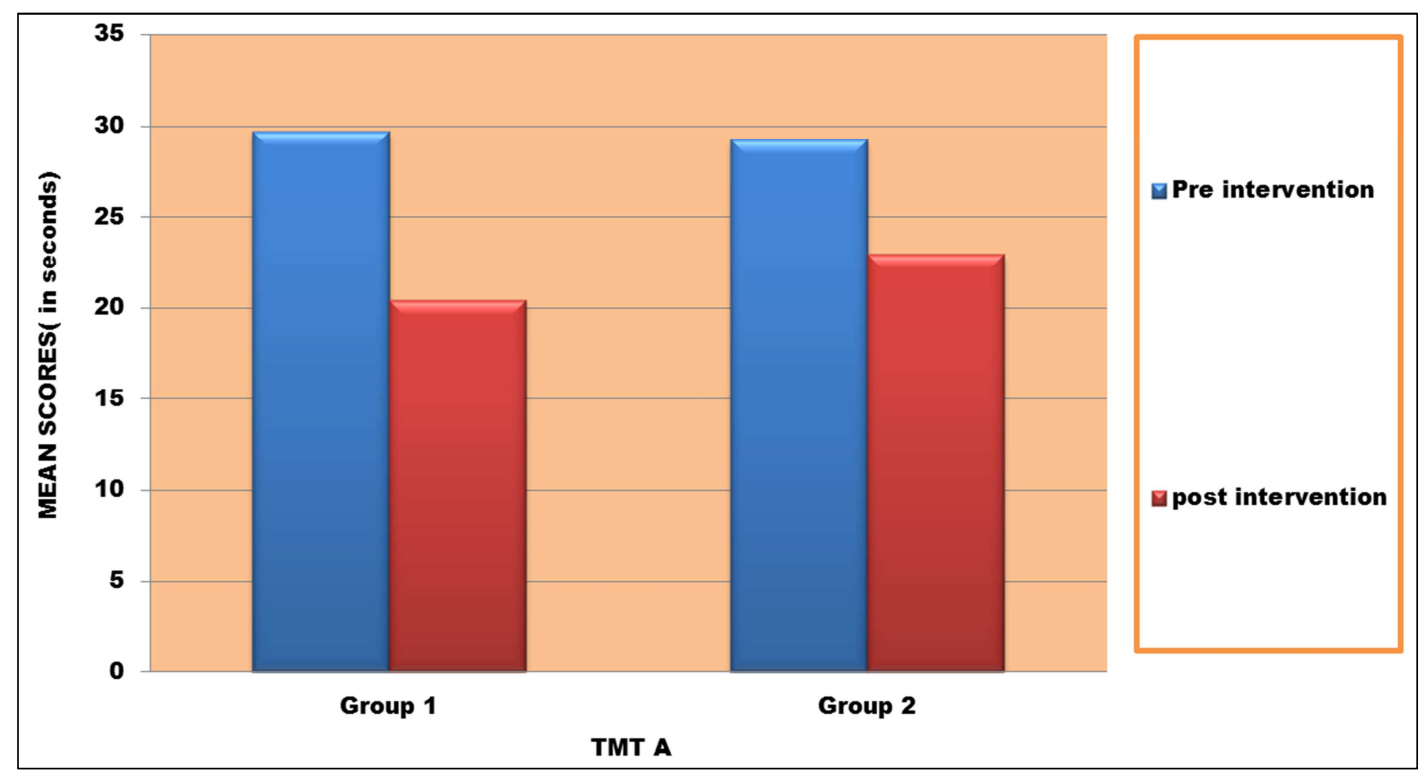

Figure 4. Comparison of Pre intervention and Post intervention in Trail Making test (Part A) of Group 1 and Group 2. 


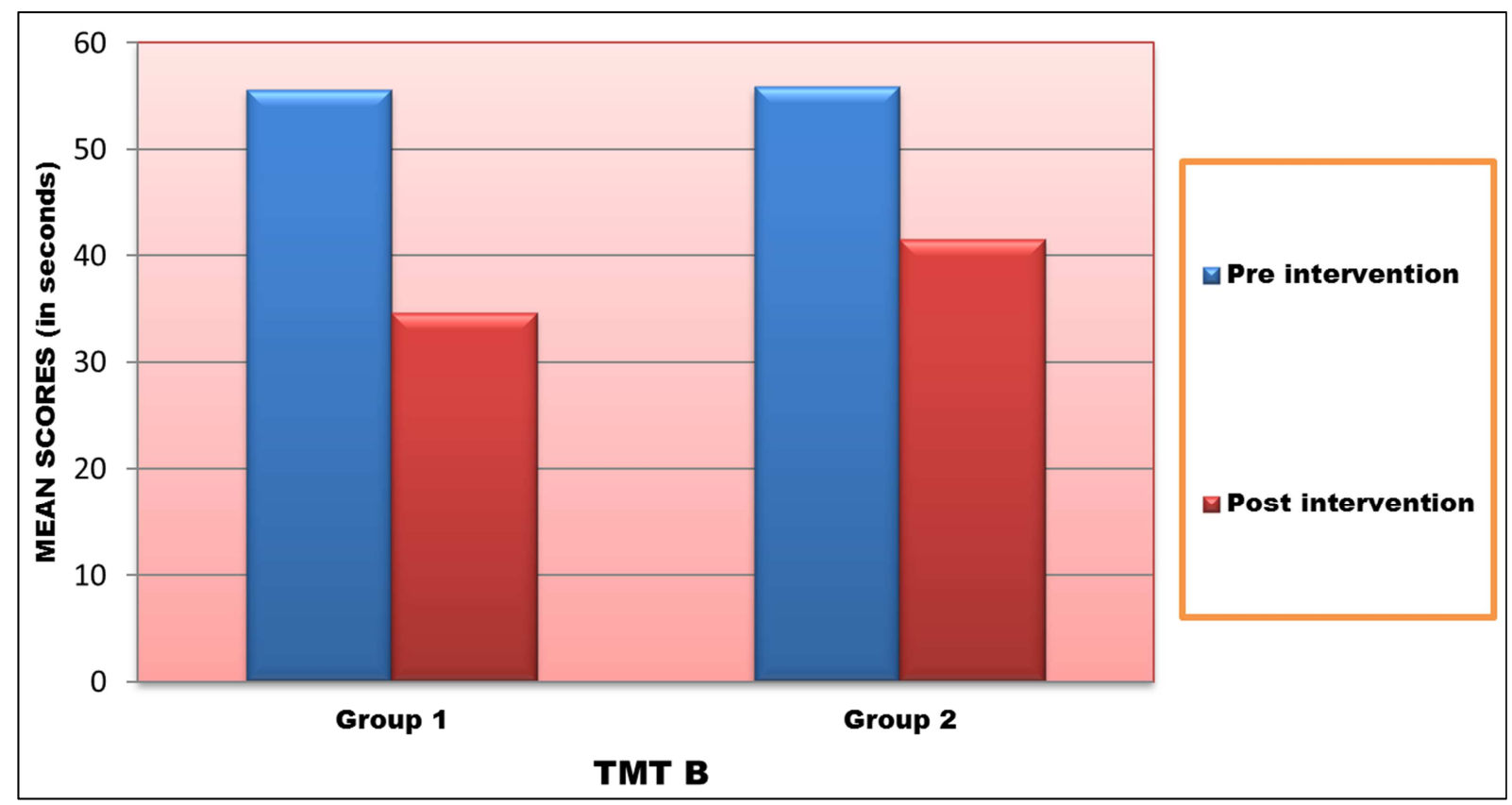

Figure 5. Comparison of Pre intervention and Post intervention in Trail Making test (Part B) of Group 1 and Group 2.

Moreover, Nintendo WII brain training games showed a significant improvement in mean difference measures of Number Comparison Test $(\mathrm{t}=2.37, \mathrm{p}=002)$ (figure 3) (Trail Making Test part $\mathrm{A}(\mathrm{t}=-2.12, \mathrm{p}=0.03)$ (figure 4) Trail Making Test part $\mathrm{B}$ $(t=-1.98, p=0.05)$ (figure 5) Backward Digit Span Test Score $(t=2.98, p=0.02)$ (figure 6) and in Perspective Taking Spatial Orientation Test $(\mathrm{t}=-2.52, \mathrm{p}=0.01)$ (figure 8$)$. However no significant difference was observed in Backward Digit Span Length $(\mathrm{t}=1.04, \mathrm{p}=0.30)$. (Figure 7). Mean and standard deviation of all variables demonstrated in (table 5).

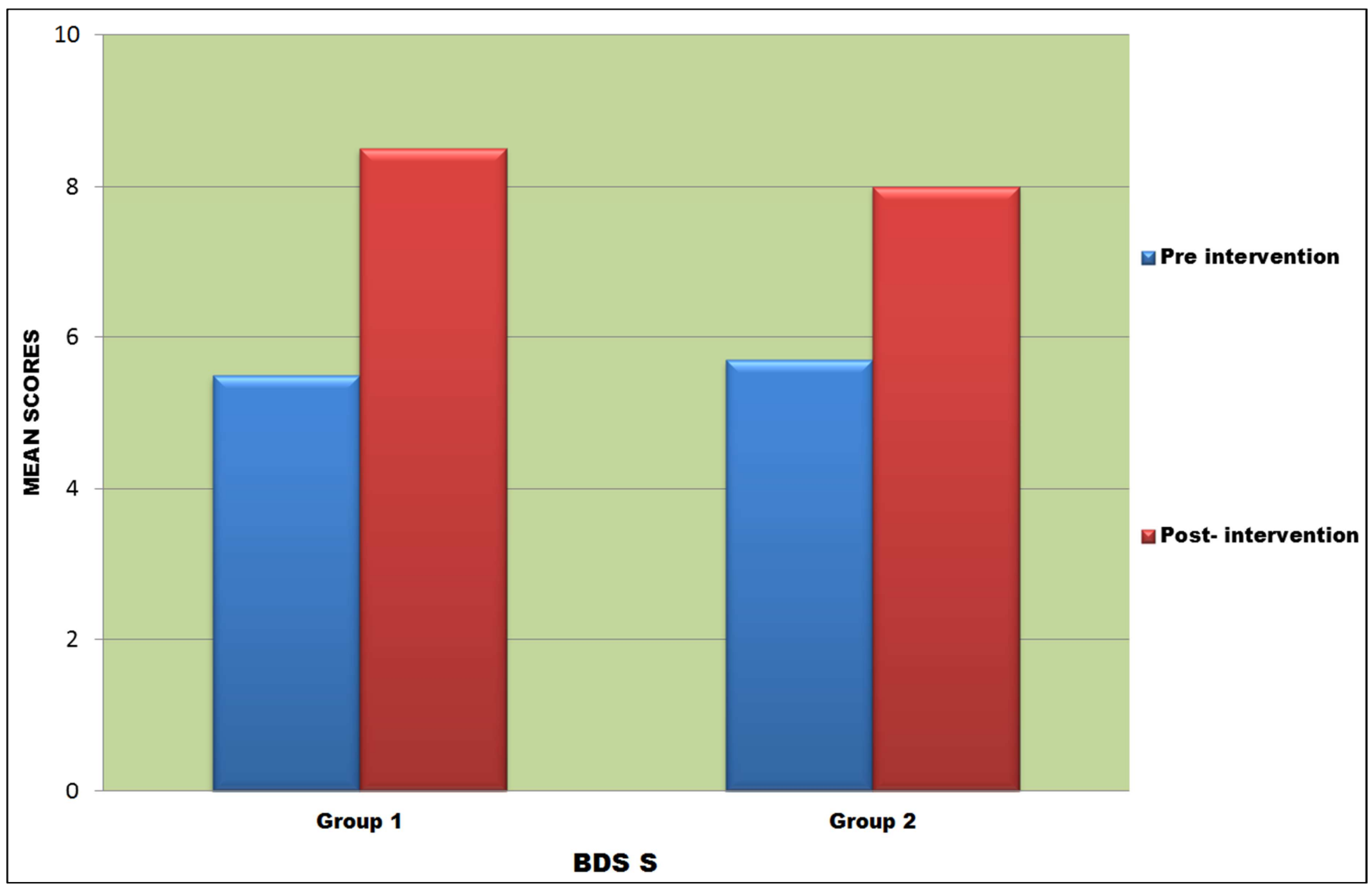

Figure 6. Comparison of Pre intervention and Post intervention in Backward Digit Span Score Measure of Group 1 and Group 2. 


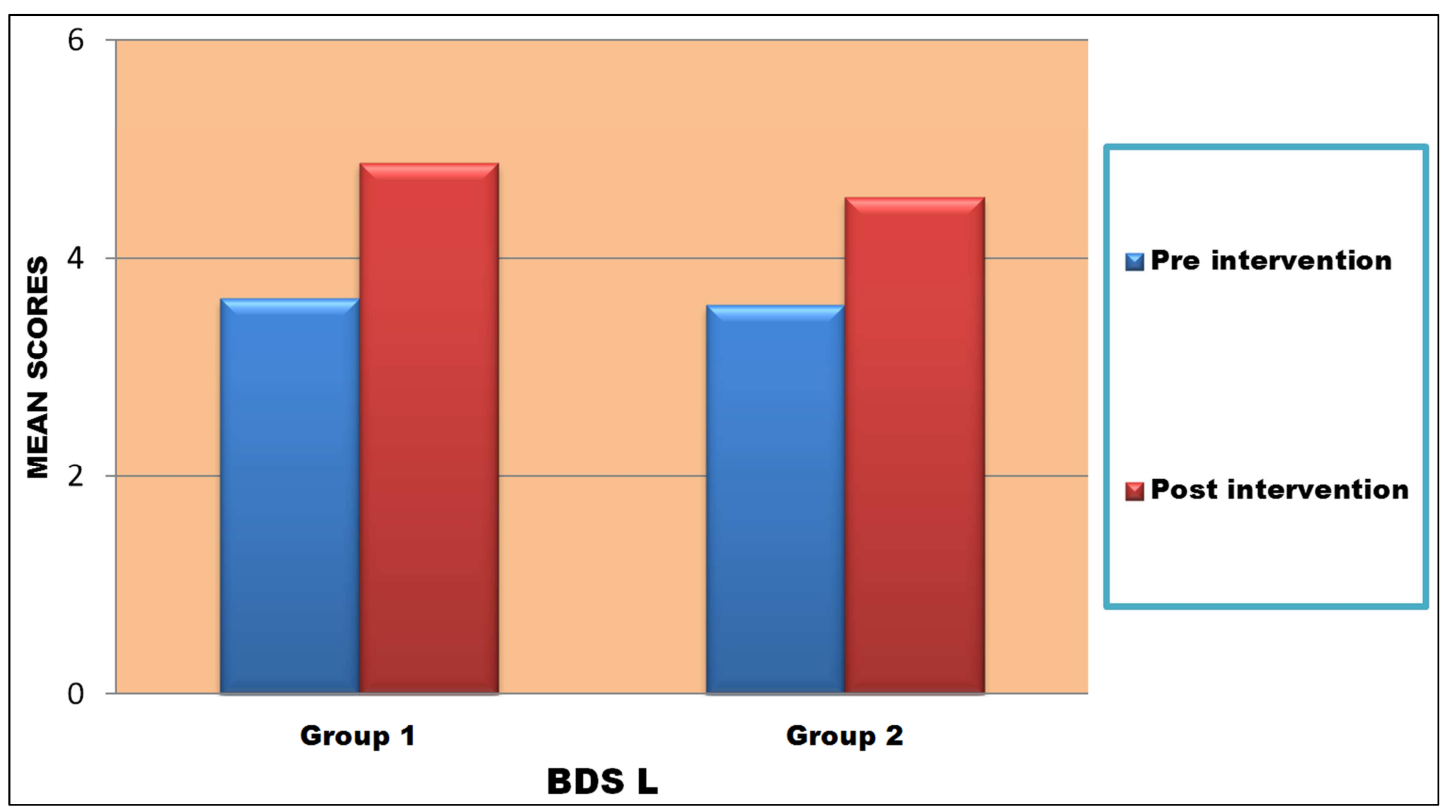

Figure 7. Comparison of Pre intervention and Post intervention in Backward Digit Span Length Measure (BDS L) of Group 1 and Group 2.

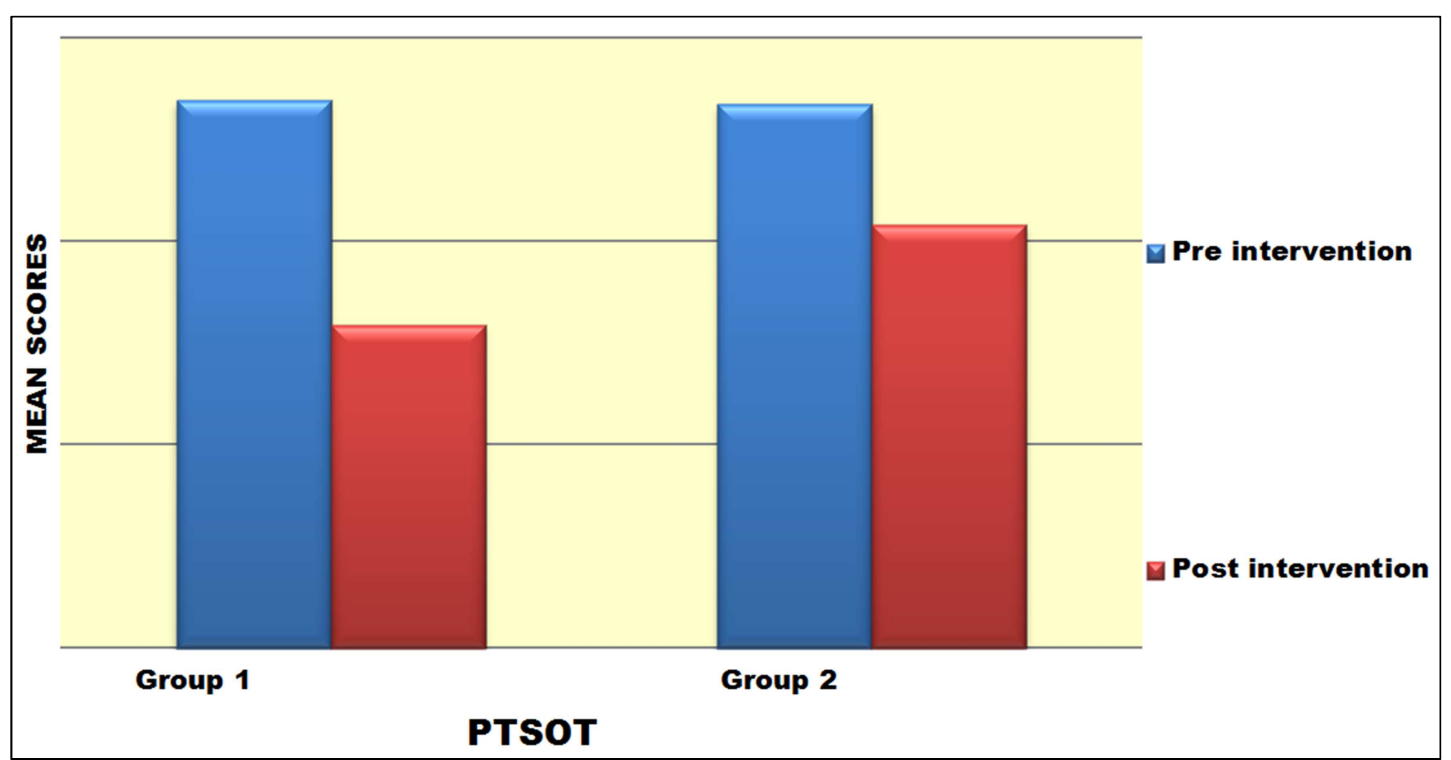

Figure 8. Comparison of Pre intervention and Post intervention in Perspective Taking Spatial Orientation Test (PTSOT) of Group 1 and Group 2.

\section{Discussion}

The results obtained after 20 treatment sessions over a period of 4 weeks with Nintendo WII big brain academy and the Nintendo WII exergames were compared for 47 subjects for perceptual speed, working memory and spatial orientation measures.

Table 3. Comparison of Pre- intervention and Post- intervention measures in Group 1.

\begin{tabular}{|c|c|c|c|c|c|c|}
\hline \multirow{2}{*}{ Variables } & \multicolumn{2}{|c|}{ Pre - intervention: Group $1(n=24)$} & \multicolumn{2}{|c|}{ Post - intervention: Group $1(n=24)$} & \multirow{2}{*}{ t value } & \multirow{2}{*}{ p value } \\
\hline & Mean & SD & Mean & SD & & \\
\hline NCT (score) & 23.50 & 7.59 & 31.04 & 7.88 & $-10.70^{*}$ & 0.00 \\
\hline TMT-A (time in sec.) & 29.69 & 6.80 & 20.44 & 5.14 & $8.58^{*}$ & 0.00 \\
\hline BDS Score & 5.50 & 1.79 & 8.50 & 2.18 & $-15.03^{*}$ & 0.00 \\
\hline BDS length & 3.63 & 1.01 & 4.88 & 1.39 & $-6.82^{*}$ & 0.00 \\
\hline PTSOT (in degrees) & 67.24 & 42.54 & 39.74 & 27.52 & $6.38^{*}$ & 0.00 \\
\hline
\end{tabular}

SD: Standard Deviation, P- Value: Level of significance, ${ }^{*}$ : Significant. 
Table 4. Comparison of Pre- intervention and Post-intervention measures in Group 2.

\begin{tabular}{|c|c|c|c|c|c|c|}
\hline \multirow{2}{*}{ Variables } & \multicolumn{2}{|c|}{ Pre - intervention: Group $2(n=23)$} & \multicolumn{2}{|c|}{ Post - intervention: Group $2(n=23)$} & \multirow{2}{*}{ t value } & \multirow{2}{*}{ p value } \\
\hline & Mean & SD & Mean & SD & & \\
\hline NCT (score) & 24.17 & 7.94 & 29.30 & 8.33 & $-7.47^{*}$ & 0.00 \\
\hline TMT-A (time in sec.) & 29.25 & 10.42 & 22.96 & 7.99 & $7.14^{*}$ & 0.00 \\
\hline TMT-B (time in sec.) & 55.94 & 25.53 & 41.52 & 16.03 & $5.79^{*}$ & 0.00 \\
\hline BDS Score & 5.70 & 1.94 & 8.00 & 2.25 & $-10.00^{*}$ & 0.00 \\
\hline BDS length & 3.57 & 1.16 & 4.57 & 1.23 & $-6.49^{*}$ & 0.00 \\
\hline PTSOT (in degrees) & 66.77 & 48.89 & 52.03 & 44.72 & $5.57^{*}$ & 0.00 \\
\hline
\end{tabular}

SD: Standard Deviation, p- Value: Level of significance, "Significant.

Table 5. Comparison of Mean Difference of variables between Group 1 and Group 2.

\begin{tabular}{|c|c|c|c|c|c|c|c|c|}
\hline \multirow[b]{2}{*}{ Variables } & \multicolumn{2}{|l|}{ Group 1} & \multicolumn{2}{|l|}{ Group 2} & \multirow{2}{*}{$\begin{array}{l}\text { Mean } \\
\text { Difference } \\
\text { Group } 1\end{array}$} & \multirow{2}{*}{$\begin{array}{l}\text { Mean } \\
\text { Difference } \\
\text { Group } 2\end{array}$} & \multirow[b]{2}{*}{ T value } & \multirow[b]{2}{*}{ P - Value } \\
\hline & $\begin{array}{l}\text { Pre intervention } \\
\text { score Mean (SD) }\end{array}$ & $\begin{array}{l}\text { Post intervention } \\
\text { score Mean (SD) }\end{array}$ & $\begin{array}{l}\text { Pre intervention } \\
\text { score Mean (SD) }\end{array}$ & $\begin{array}{l}\text { Post intervention } \\
\text { score Mean (SD) }\end{array}$ & & & & \\
\hline NCT (score) & $23.50(7.59)$ & $31.04(7.88)$ & $24.17(7.94)$ & $29.30(8.33)$ & 7.42 & 5.13 & $2.37^{*}$ & 0.02 \\
\hline TMT A (in sec.) & $29.69(6.80)$ & $20.22(5.41)$ & $29.25(10.42)$ & $22.96(7.99)$ & -9.25 & -6.29 & $-2.12^{*}$ & 0.03 \\
\hline TMT B (in sec) & 55.65 (18.89) & $34.54(9.72)$ & $55.94(25.53)$ & $41.52(16.03)$ & -21.11 & -14.41 & $-1.98^{*}$ & 0.05 \\
\hline BDS S & $5.50(1.79)$ & $8.50(2.18)$ & $5.70(1.94)$ & $8.00(2.25)$ & 3.00 & 2.30 & $2.98^{*}$ & 0.02 \\
\hline BDS L & $3.63(1.01)$ & $4.88(1.39)$ & $3.57(1.16)$ & $4.57(1.23)$ & 1.25 & 1.00 & $1.04^{\mathrm{NS}}$ & 0.30 \\
\hline PTSOT (in degree) & $67.24(42.54)$ & $39.74(27.52)$ & $66.77(48.89)$ & $52.03(44.72)$ & -27.50 & -14.73 & $-2.52 *$ & 0.01 \\
\hline
\end{tabular}

SD: Standard Deviation, p- Value: Level of significance, ${ }^{*}$ Significant, NS: Non- significant.

The findings of the study revealed that the subjects in Group 1 receiving Nintendo WII brain training games significantly improved in the scores of Number comparison test, Trail Making Test- part A and B, Backward Digit Span Test and Perspective Taking Spatial Orientation Test after intervention. These findings are well supported by the previous work of Ackerman, Kanfer and Calderwood (2010) [17], who have used a crossover design, where each participant took part in both the training methods- the WII big brain academy and a reading article session. However, the study showed a significant improvement in all measures of perceptual speed ability tests and two of the general fluid intelligence tests in middle age adults. Another study by Baniqued et al (2014) [30] also showed improvements in trail making test part A and B, spatial working memory, running span and symmetry span measures after 15 hours of training with a casual video game in young adults.

The subject's in Group 2 who were trained with the Nintendo WII Exergames also revealed a significant improvement in the scores of Number comparison test, Trail making test- part A and B, Backward digit span test and Perspective taking spatial orientation test. The result supports the study by Pauline Maillot, Perrot and Hartely (2012) [9] that assessed the potential of exergame training - The Nintendo WII-Fit and sports game as a mode of physical activity that have a cognitive benefit for older adults. The result showed a significant improvement in all executive functioning, processing speed measures especially trail making test and number comparison test but not on the visuospatial measures.

Furthermore, the present results demonstrated that the subjects trained with Nintendo WII brain training games significantly improved their perceptual speed, working memory and spatial orientation in young adults than those trained using Nintendo WII exergames. Similar findings were seen by Rui Nouch et al (2013) [19], who compared the brain training games with a non brain training games for executive function, working memory and processing speed. The study showed a significant improvement in some of the measures of executive functions, working memory and processing speed after playing Nintendo WII brain training game- the brain age for 15 minutes per day for 5 times a week for 4 weeks. However, both the groups failed to improve backward digit span forward and backward score, raven advance progressive matrix score and the measure of reading ability. The possible reason for this could be the short duration of 15 minutes game play that was not sufficient to improve the measures, a relative small sample size or may be the use of a brain training game which is not proven for a specific training domain.

In our study, Nintendo WII brain Training game did not show a significant improvement in backward digit span length measure when compared to Nintendo WII exergame group. A possible reason for this could be that the test- retest reliability of the score measure is better than the span length measure as reported by Gloria S. Walter (2003) [28].

Significant improvement in spatial orientation was seen in subjects trained using brain training game as compared to those trained with exergames. The findings are similar to the study by Chien Heing (2014) [12] which uses a domain related designed computer game called a treasure hunting game. The study showed a significant improvement in perspective taking spatial orientation test (PTSOT) and Guilford Zimmeran spatial orientation test (GZOT) and also in Corsi block tapping test, a measure of spatial memory domain with the single session of 45-50 minutes as compared to control group in children (12-13 years).

The current study presents several strengths as its training regimes fulfill the principles of learning and learning transfer as compared to the previous studies. All the determinants of 
learning as described in the study by Green C. S (2008) [32] can be well explained with the study. For instance task difficulty- the principal of utilizing small increases in task difficulty is implicit in nearly every videogame. As the players progress through levels they learn new skills and techniques that allow player to master circumstances. In the present study participants moved to the next level of difficulty during training, only when they have demonstrated a sufficient mastery of their current level i.e. getting score of $300+$ and a gold medal in easy level of Nintendo WII Big brain academy and getting four stars in beginner level of Nintendo WII Exergaming group.

The other determinant of learning is motivation and arousal. Motivation is critical component of most major theories of learning. Vygotsk's (1978) “zone of proximal development" theory corresponds well with the skill learning literature. According to the theory, motivation is highest and learning is most efficient when tasks are made just slightly difficult, yet still doable. Arousal is at the heart of many learning theories in social science. Arousal is often thought of an abstract construct encompassing a variety of processes including those hat mediate alertness and wakefulness and has been defined in terms of autonomic responses (e.g. change in heart rate, pupil dilation), neurophysiological response (activity of reticular system, dopamine) and/or behavioral responses (increased alertness). Videogames are known to strongly elicit both autonomic and neurophysiological responses.

Last but not the least, another possible determinant of learning fulfilled by the current study is the feedback. The games in the current study provide both visual and auditory feedback during the game play and at the end of each game in both the training groups.

Thus, the nature of the game training in the study can possibly be the reason of significant improvement in both the groups as it fulfills all the principles needed for skill acquisition, learning and learning transfer.

\section{Clinical Implications}

Perceptual speed, Working Memory and Spatial Orientation, the domains of fluid intelligence begins to decline starting from early 20's. This study has helped in identifying the effectiveness of Nintendo WII brain training games and Nintendo WII exergames as a training method in improving perceptual speed, working memory and spatial orientation in young adults. The study does not state that every young adult should engage in gaming. However, the study provides the evidence that commercial brain training games as well as exergames might be a simple, convenient means to improve some of the cognitive functions.

\section{Limitations}

1. Study considered only three cognitive domains the perceptual speed, working memory and spatial orientation.
2. Long term benefits were not assessed in this study

3. Far transfer effects i..e transfer to untrained tasks are not considered in the study

4. The subjective feeling of participants i..e motivation, satisfaction, fatigue were not taken into account.

\section{Future Directions}

One of the important future directions is to examine whether or not brain training games can be expected to have long term beneficial effects on cognitive functions. Other aspects of cognition such as attention, long term memory can also be trained using the Nintendo WII brain training games. The protocol can be used for children, older subjects, neurological deficit patients such as Cerebral Palsy, stroke, attention deficits.

\section{Conclusion}

The results of the study proved that both Nintendo WII brain training games and Nintendo WII exergames have a great potential to improve cognitive abilities in young adults as both showed a significant difference in the scores of Number Comparison Test, Trail Making Test (Part A and Part B), Backward Digit Span Test (score and length measure) and Perspective Taking Spatial Orientation Test after intervention.

When compared between the groups Nintendo WII brain training games emerge out as a more efficient mode for training perceptual speed, working memory and spatial orientation in young adults. However, no significant improvement was observed in backward digit span length measure.

Thus, concluding that there is a significant difference found in perceptual speed, working memory and spatial orientation among the subjects who were trained with Nintendo WII brain training games as compared to the subjects who were trained with Nintendo WII exergames. Hence, our experimental hypothesis stating that Nintendo WII brain training games are more efficient than Nintendo WII exergames in improving perceptual speed, working memory and spatial orientation in young adults stands true and the null hypothesis is rejected.

\section{References}

[1] Sacco RG. Re-envisaging the eight developmental stages of ERIK ERIKSON: the Fibonacci life chart method FLCM. Journal of Educational and Developmental Psychology. 2013; Vol. 3, No. 1: 140-146.

[2] Salthouse TA. Consequences of age related cognitive decline. Annul. Rev. Psychol. 2011; Vol. 63: 5.1-5.26.

[3] Harada CN, Natelson MC, Triebel KL. Normal cognitive aging. Clinc Geratr Med. 2013; Vol. 29: 737-752.

[4] Salthouse TA. When does age related cognitive decline begin? Neurobiolo Aging. 2009; Vol. 30, No. 4: 507-514. 
[5] Salthouse TA. What and when of cognitive aging. Current directions in psychological science. 2004; Vol. 13, No. 4: 140144.

[6] Jaeggi SM, Buschkuehl M, Jonides $\mathrm{J}$ and Perring WJ. Improving fluid intelligence with training on working memory.

[7] Borson S. Cognition, aging and disabilities: conceptual issues. Phys Med Rehabil Clinc N Am. 2010; Vol. 21: 375-381.

[8] Jackson R. C, Farrow D. Implicit perceptual training: how, when and why? Human movement science. 2005; Vol. 24: 308-32.

[9] Maillot P, Perrot A and Hartley A/ Effect of interactive physical activity videogame training on physical and cognitive function in older adults Psychology and aging, American Psychological Association 2012.

[10] Takeuchi H, Taki Y, Kawashima R. Effect of working memory training on cognitive functions and neural systems. Reviews in Neurosciences. 2010; Vol. 21: 427-449.

[11] Yuan K, Streedle J, Shavelson R, Alonzo A, Oppezzo M. Working memory, fluid intelligence and science learning. Educational Research Review. 2006: 83-98.

[12] Lin $\mathrm{CH}$, Chen CM, Lou YU. Developing spatial orientation and spatial memory with a treasure hunting game. Educational Technology and Society. 2014; Vol. 17, No. 3: 79-82.

[13] Shwetha B and Sudhakar HC. Influence of shift work on cognitive performance in male business process outsourcing employees. Indian J. Occup Environ Med. 2012; Vol. 16, No. 3: $114-118$.

[14] Sunitha S, Gururaj G. Health behavior and problem among young people in India: cause for concern and call for action. Indian J. Med Res. 2014; 140: 185-208.

[15] Gligoroska JP et al. Assessment of working memory capacity in young healthy adults with different level of long term physical activity. Research gate. 2010: 132-137.

[16] Tanaka et al. A Comparison of Exergaming Interfaces for Use in Rehabilitation Programs and Research; Vol 6 (9): 69-81.

[17] Ackerman PL, Kerifer R and Colder wood C. Use it as loose it? WII brain exercise practice and reading for domin in knowledge. Psychol Aging 201025 (4): 753-766.

[18] Nouchi R and Kawashima R. Improving cognitive function from children to old age. S systematic review of recent smart ageing intervention studies. Advances in Neurosciences. 2014: $1-15$.
[19] Nouchi R et. Al. Brain training game boosts executive function, working memory and processing speed in the young adults. A parodied controlled trial. PLOS one 2013; Vol 8 Issue 2: 1-13.

[20] Gluhm S et al. Cognitive performance on mini-mental state examination and the montreal cognitive assessment across healthy adults lifespan. Cog Behav Neurol. 2013; 26 (1): 1-11.

[21] Kumar AM, Saraswathi I, Sembulingam P. Impact of perceived stress on cognitive ability and physical performance in young adults. World journal of pharmaceutical research. 2015; Vol. 4, Issue 10: 1620-1632.

[22] Segal DL, Colidge FL, Cahalill BS and Reiley AO. Psychometric properties of Beck Depression Inventory II among community dwelling older adults. Behaviour Modification. 2008; 32 (1): 3-20.

[23] Nintendo WII big brain academy instructional booklet.

[24] Ackerman PL, Beier ME. Further exploration of perceptual speed abilities in the contextof assessment methods, cognitive abilities and individual differences during skill acquisition. Journal of Experimental Psychology: Applied. 2007; Vol. 13, No. 4: 249-272.

[25] Ekstrom RB, French JW, Harman HH, Dermen D. Manual for kit of factor -referenced cognitive test. 1976: 184-186.

[26] Tombaugh T. N. Trail making test A and B: normative data stratified by age and education. Archives of clinical neuropsychology. 2004; Vol. 19: 203-214.

[27] Mihalik J. Age related differences and reliability on computerized and paper and pencil neurocognitive assessment batteries. Journal of athletic training. 2012; 47 (3): 297-305.

[28] Waters G. S. The reliability and stability of verbal working memory measures Behavior Research methods, Instruments and computers 2003; 35 (4): 550-564.

[29] Hegarty M and Wasler D. A dissociation between mental rotation and perspective taking spatial ability. Intelligence 2004; 32: 175-191.

[30] Baniqad PL. et al. Cognitive training with casual videogames: Points to consider Frontiers In Psychology: 2014; Articles 41 19.

[31] Green CS and Bavelior D. Exercising Your Brain: A Review of Human Brain Plasticity and Training-Induced Learning. Psychol Aging. 2008; 23 (4): 692-701. 